\title{
Fabrication of Replica Mold by Room Temperature Nanoimprinting using Organic Spin-on-glass
}

\author{
Makoto Okada $^{1,3,4}$, Masatoshi Maeda ${ }^{2}$, Satoshi Shimatani ${ }^{2}$, \\ Shoji Otaka ${ }^{2}$, and Shinji Matsui ${ }^{1,3}$ \\ ${ }^{I}$ Graduate School of Science, LASTI, University of Hyogo \\ 3-1-2 Koto, Kamigori, Ako, Hyogo 678-1205, Japan \\ ${ }^{2}$ Tokyo Ohka Kogyo Co., Ltd., 150 Nakamaruko, Nakahara, Kawasaki 211-0012, Japan \\ ${ }^{3} J S T$-CREST, Sanbancho, Chiyoda-ku, Tokyo, 102-0075, Japan \\ ${ }^{4}$ JSPS, 6 Ichibancho, Chiyoda-ku, Tokyo 102-8471, Japan \\ E-mail:m.okada@lasti.u-hyogo.ac.jp
}

Keywords: room temperature nanoimprinting, organic spin-on-glass, replica mold

\section{Introduction}

Nanoimprint lithography (NIL) can transfer the nano-patterns on the master mold into nanoimprint resin by physical contact. [1-3] It can be used effectively to fabricate nano-patterns with simple process and low cost and is therefore considered to be one of the most promising next-generation nano-patterning technologies.

A mold replication process with easy and low-cost is required to prevent the damage to a master mold. So far, the duplication of polymer molds from the master mold has been reported. [4] The polymer mold is also required a high mechanical strength because the pattern deformation of the polymer mold with a low mechanical strength is induced by a NIL with a high pressure.

Hydrogen silsesquioxane (HSQ) is a type of spin-on-glass material. As a technique to fabricate the nanostructure of the HSQ, room temperature (RT) NIL is reported. [5-8] The resin-thermal cycle and UV exposure is not needed in the RT-NIL, as shown in Fig. 1. However, we must apply a high pressure to the HSQ in this process. To reduce the imprinting pressure, the organic SOG $(\mathrm{O}-\mathrm{SOG})$ is produced from Tokyo Ohka Kogyo Co.. Although the O-SOG has the organofunctional groups, the main chain consisted of -Si-O- bond. We therefore assume that the O-SOG has a higher mechanical strength than the polymer.

(1)
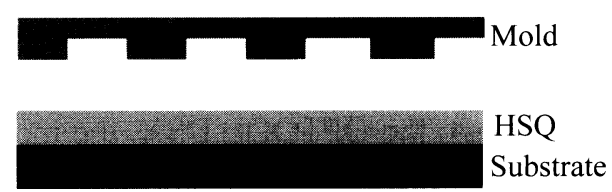

(2) Pressing

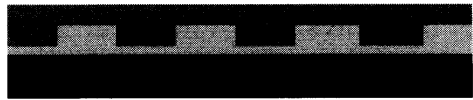

(3)

Demolding

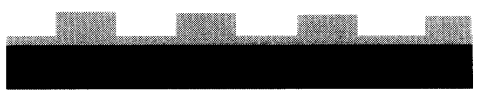

Fig. 1 Schematic of room temperature nanoimprint process.

In this study, we fabricated the replica mold of the O-SOG by RT-NIL and demonstrated the thermal nanoimprinting using the replica mold. 


\section{Replica mold fabrication using O-SOG}

$\mathrm{A} \mathrm{SiO}_{2} / \mathrm{Si}$ mold was fabricated by electron beam (EB) lithography and reactive ion etching (RIE). Figure 2 shows the scanning electron microscopy (SEM) image of the line and space (L\&S) pattern on the mold. The line- and space-width and height were 190, 300, and $150 \mathrm{~nm}$, respectively. The L\&S pattern area was $3.6 \mathrm{~mm}$ square on the mold.

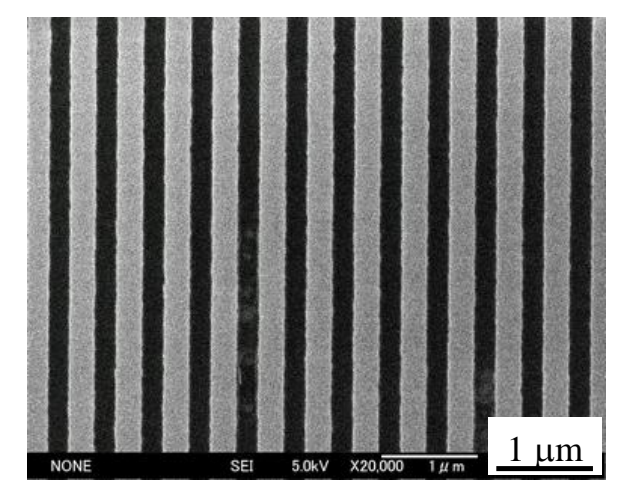

Fig. 2 SEM image of L\&S pattern on mold.

This mold was coated an antisticking layer by a dip coating. We used a silane-coupling agent with fluoropolymer (OPTOOL DSX: Daikin Industries) as the antisticking layer. [9] The dip coating process is as follows; (1) The mold is dipped into the 0.1 weight $\%$ OPTOOL DSX diluted by HD-TH (Daikin Industries) for 1min at room temperature in air atmosphere. (2) After dipping into the OPTOOL DSX, the mold is left in the high humidity atmosphere for $1 \mathrm{~h}$. (3) Then, to remove the multiple layers, the mold is rinsed by HD-TH for $5 \mathrm{~min}$.

We used OCNL540 (Tokyo Ohka Kogyo Co.) as the O-SOG. Figure 3 shows the chemical structure of O-SOG. This O-SOG has a methyl group in the polymer structure instead of the $\mathrm{H}$ group in the SOG. The curing speed of the OCNL540 is reduced by controlling the sol-gel reaction. The low curing speed induce to the low imprinting pressure.

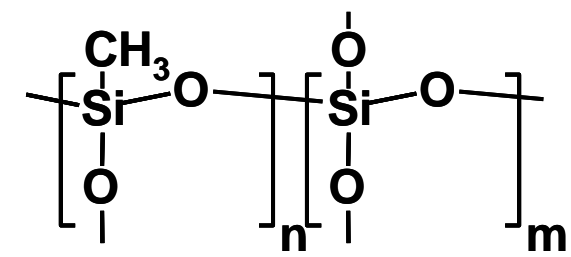

Fig. 3 Chemical structure of OCNL540.

We fabricate the replica mold for thermal nanoimprinting by RT-NIL using O-SOG. The O-SOG was spin-coated with $2000 \mathrm{rpm}$ on the $15 \mathrm{~mm}$ square $\mathrm{SiO}_{2} / \mathrm{Si}$ substrate. The O-SOG film thickness was about $200 \mathrm{~nm}$ in this case. We used the NM-0901SQ (MEISYO KIKO Co.) as a nanoimprinting system. The imprinting pressure and time were $15 \mathrm{MPa}$ and 2 min, respectively. Figure 4(a) shows the SEM image of the L\&S pattern on the O-SOG. The pattern was clearly imprinted

To demonstrate thermal nanoimprinting using the O-SOG replica mold, the replica mold was coated with the antisticking layer by above dip coating process. However, the imprinted O-SOG pattern was disappeared after leaving in the high humidity atmosphere. We assumed from this result that the imprinted O-SOG pattern was removed by the water because the O-SOG was not perfectly cured by RT-NIL. We therefore tried to dip the imprinted $\mathrm{O}-\mathrm{SOG}$ pattern into the water.

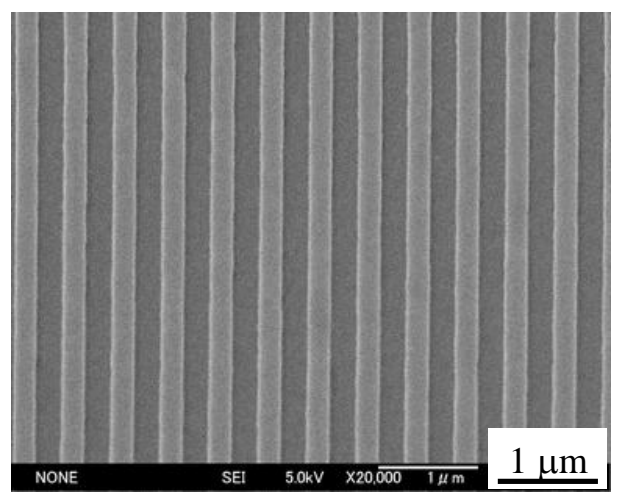

(a)

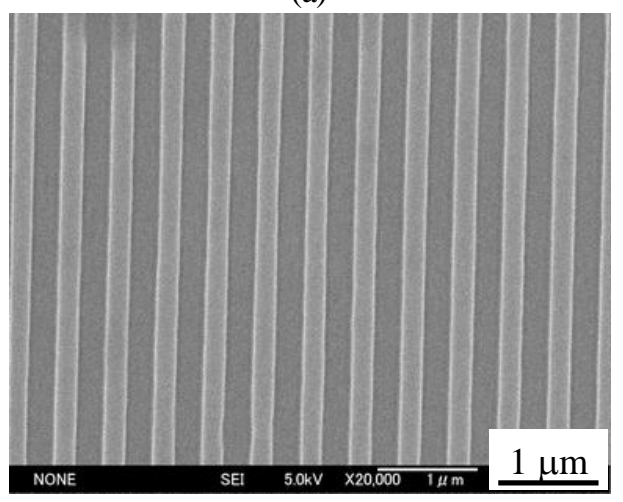

(b)

Fig. 4 SEM image of (a) imprinted O-SOG pattern. (b) post-baked O-SOG pattern.

As the result, the O-SOG pattern and layer were clearly removed. This indicates that the imprinted O-SOG pattern must be cured before the dip coating process of the antisticking layer. To resolve this issue, 
we carried out post-baking at $120{ }^{\circ} \mathrm{C}$ for $2 \mathrm{~min}$. The pattern shrinkage was not occurred by this baking process, as shown in Fig. 4(b). We confirmed that the post-baked O-SOG pattern was maintained after dipping into the water. This result indicates that the O-SOG was cured by post-baking. In fact, we were able to carry out the dip coating process of the antisticking layer for the post-baked O-SOG replica mold.

\section{Thermal nanoimprinting using O-SOG replica mold}

We next demonstrated the thermal nanoimprinting using the O-SOG replica mold. NEB-22 (Sumitomo Chemical Co.) was used as a thermal nanoimprint resin. The NEB-22 was spin-coated on the Si substrate and, then, this substrate was prebaked at $110{ }^{\circ} \mathrm{C}$ for 2 $\mathrm{min}$. The mold and substrate temperatures increased by $150{ }^{\circ} \mathrm{C}$ during thermal nanoimprinting. The imprinting pressure and time were $20 \mathrm{MPa}$ and $60 \mathrm{sec}$, respectively. After cooling process, the mold was demolded from the NEB-22/Si substrate. The L\&S pattern was successfully transferred onto the NEB-22, as shown in Fig. 5(a).

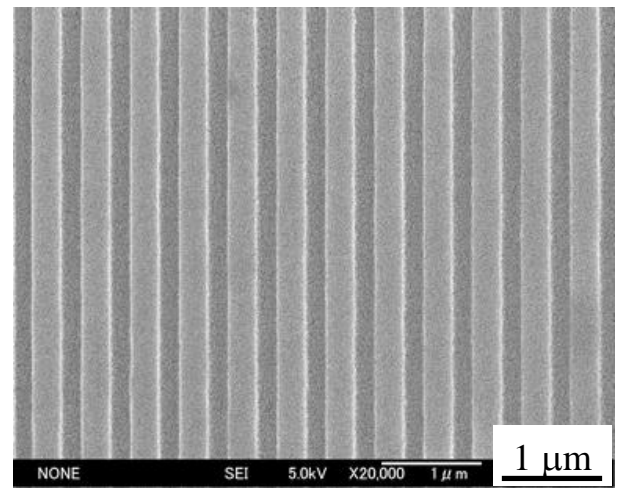

(a)

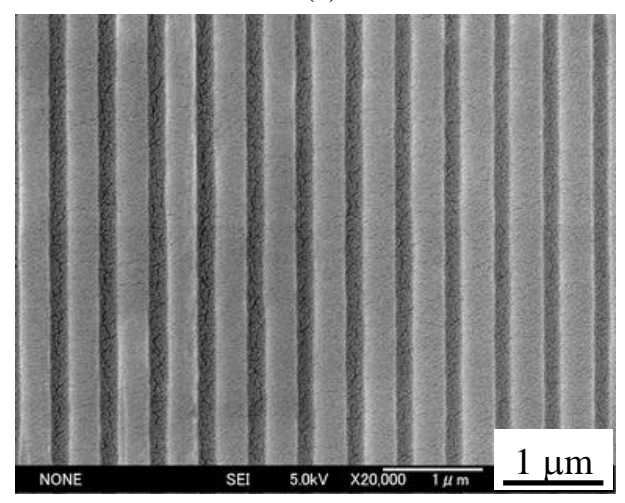

Fig. 5 SEM image of (a) imprinted NEB-22 pattern by thermal nanoimprinting using O-SOG replica mold.

(b) 40th imprinted NEB-22 pattern by step and repeat thermal nanoimprinting.
In the nanoimprinting process, the adhesion force between the O-SOG and $\mathrm{SiO}_{2} / \mathrm{Si}$ substrate is an important factor because the replica mold must be in direct contact with the resin. If this force is small, the O-SOG pattern was separated from the substrate during nanoimprinting process. To evaluate the adhesion force between the O-SOG and $\mathrm{SiO}_{2} / \mathrm{Si}$ substrate, we demonstrated step and repeat thermal nanoimprinting using the O-SOG replica mold. The imprinting pressure was $20 \mathrm{MPa}$. The mold and substrate temperatures were 180 and $90{ }^{\circ} \mathrm{C}$, respectively. Figure 5(b) shows the SEM image of the 40th imprinted pattern. The pattern was clearly imprinted on the NEB-22. Furthermore, after 40 times of repeated nanoimprinting, the O-SOG pattern was not separated from the $\mathrm{SiO}_{2} / \mathrm{Si}$ substrate. We confirmed from this result that the adhesion force between the O-SOG and $\mathrm{SiO}_{2} / \mathrm{Si}$ substrate is sufficient to carry out the thermal nanoimprinting.

\section{Conclusion}

We proposed the replica mold fabrication using the O-SOG. The pattern was imprinted on the O-SOG with RT-NIL. We demonstrated thermal nanoimprinting using the O-SOG replica mold with the antisticking layer. The pattern was successfully imprinted on the NEB-22. In addition, we were able to carry out 40 times of repeated thermal nanoimprinting using the O-SOG replica mold.

\section{References}

1) S. Y. Chou, P. R. Krauss, and P. J. Renstrom: Appl. Phys. Lett. 67 (1995) 3114.

2) S. Y. Chou, P. R. Krauss, and P. J. Renstrom: Science 272 (1996) 85.

3) S. Y. Chou, P. R. Krauss, W. Zhang, L. Guo, and L. Zhuang: J. Vac. Sci. Technol. B 15 (1997) 2897.

4) Y. Kawaguchi, F. Nonaka, Y. Sanada: Microelectronic Engineering, 84 (2007) 973.

5) S. Matsui, Y. Igaku, H. Ishigaki, J. Fujita, N. Ishida, Y. Ochiai, M. Komuro, and H. Hiroshima: J. Vac. Sci. Technol. B 19 (2001) 2801.

6) S. Matsui, Y. Igaku, H. Ishigaki, J. Fujita, N. Ishida, Y. Ochiai, H. Namatsu, M. Komuro, and $H$. Hiroshima: J. Vac. Sci. Technol. B 21 (2003) 688.

7) K. Nakamatsu, K. Watanabe, K. Tone, T. Katase, W. Hattori, Y. Ochiai, T, Matsuo, M. Sasago, H. Namatsu, M. Komuro, and S. Matsui: Jpn. J. Appl. Phys. 43 
(2004) 4050 .

8) K. Nakamatsu, K. Watanabe, K. Tone, H. Namatsu, and S. Matsui: J. Vac. Sci. Technol. B 23 (2005) 507.
9) Y. Hirai, S. Yoshida, A. Okamoto, Y. Tanaka, M. Endo, S. Irie, H. Nakagawa, and M. Sasago: $J$. Photopolym. Sci. Technol., 14 (2001) 457 